\title{
Porocéphalose à Nettorhynchus (Armillifer) armillatus (Wyman, 1845) chez un chat
}

\author{
par S. GRÉTILLAT et G. THIÉRY
}

L'autopsie d'un chat abattu en juin 1960 au service d'anatomie pathologique du Laboratoire central de l'élevage à Dakar, nous permet de signaler un cas de porocéphalose féline.

\section{Commémoratifs}

Une chatte âgée d'environ deux ans, ayant mordu un enfant, est apportée au laboratoire pour observation. Cet animal, ayant mis bas récemment, n'a manifesté aucun trouble pendant les trois semaines d'observation qui ont précédé sa mise à mort.

Elle vivait dans une villa de Dakar-Hann proche du parc forestier. Cette localisation est à considérer, ce parc constituant une réserve de serpents (bitis, couleuvres diverses, najas) qui émigrent dans les environs, principalement pendant la saison des pluies. Toutefois, l'abondance des reptiles n'est que relative.

\section{Anatomie pathologique}

A l'ouverture des grandes cavités du cadavre, on était frappé par la présence d'un grand nombre de kystes de Pentastomidae (ordre Acarina). Ceux-ci au nombre d'une cinquantaine, étaient particulièrement nombreux dans la cavité abdominale où ils étaient fixés à l'épiploon, au mésentère, à la surface de la rate et du foie, ainsi qu'à la paroi abdominale. Quatre parasites avaient envahi la cavité thoracique, répartis sur le médiastin (2), sur la face antérieure du diaphragme (1) et la paroi thoracique (1).

Tous ces parasites siégeaient en position

Reçu pour publication août 1960

Rev. Elev. Méd. vét. Pays trop. 1960, 13, no 4. superficielle, sous-séreuse. Aucun d'eux n'était intra-hépatique ou intra-splénique.

L'étude des coupes histologiques de la rate et du foie, intéressant les kystes parasitaires, ne révèle pas de réaction inflammatoire périphérique. Le kyste est limité par une fine membrane collagène hyaline qui s'unit au tissu conjonctif avoisinant. Lorsque plusieurs kystes sont jointifs, il existe une faible réaction collagène d'union.

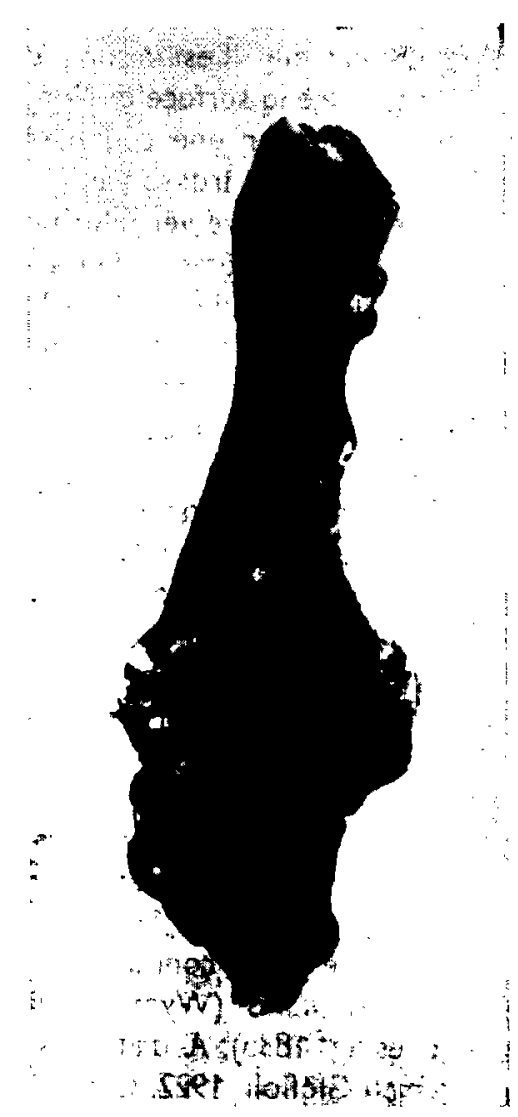

Fıg. 1. - Rate de chat avec nombreux porocéphales. 


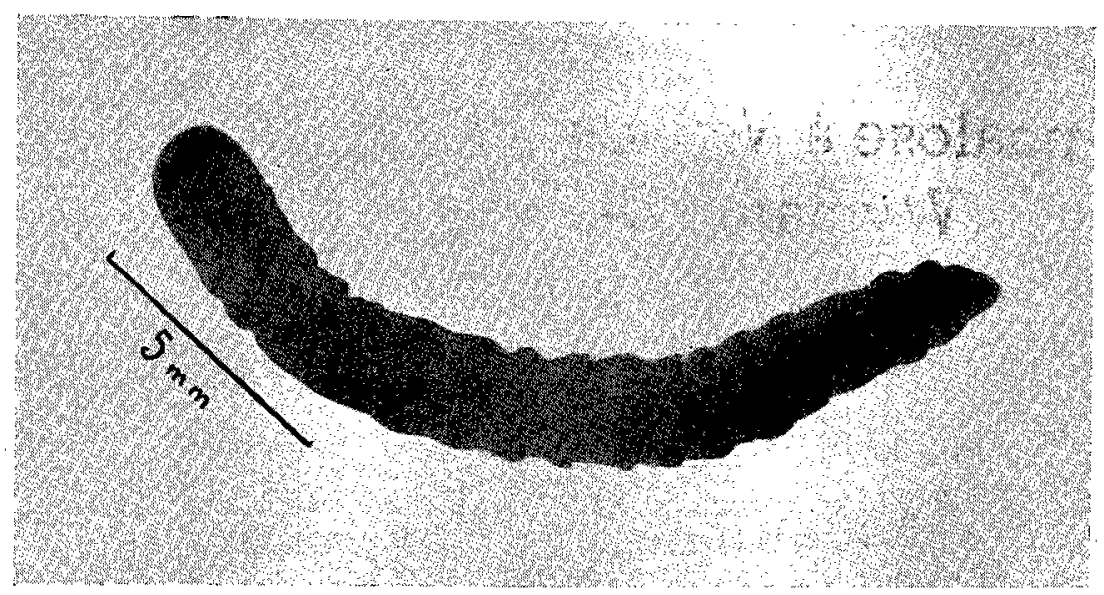

Fig. 2. - Nymphe de Neftorhynchus (Armillifer) armillatus désenkystée et montée après éclaircissement.

\section{Description sommaire du kyste et de la larve se trouvant à son intérieur}

Le kyste (fig. no 1) a un diamètre de 6 à $9 \mathrm{~mm}$ et 2 à 2,5 mm d'épaisseur. Il est arrondi et appliqué directement contre la surface de l'organe ou de la séreuse. Limité par une coque réactionnelle conjonctive mince et transparente, on distingue d̀ l'intérieur une larve vermiforme, annelée et enroulée sur elle-même. Libérée de son enveloppe et déroulée ensuite (fig. $n^{\circ} 2$ ), elle a une longueur de 11 à $18 \mathrm{~mm}$ pour une épaisseur de $2 \mathrm{~mm}$ environ. Son corps qui est cylindrique présente 18 à 24 anneaux suivant les exemplaires.

Après éclaircissement, les appendices céphalothoraciques (quatre crochets simples), alignés avec un cadre buccal arrondi, apparaissent sur la surface ventrale de la partie antérieure hémisphérique (fig. no 3). La disposition des crochets et la forme du cadre buccal permettent de ranger nos exemplaires dans le genre Armillifer Sambon, 1922.

Du genre Armillifer, mis en synonymie avec Nettorhynchus P. Gervais, 1946 (Nettorhynque de Blainville, 1824) par Dollfus en 1950, et dont six espèces sont décrites, on connaît les formes nymphales d'A. armillatus (Wyman, 1835), A. moniliformis (Diesing, 1835), A. annulatus (Baird, 1853) et A. Brumpti Giglioli, 1922. Cette dernière espèce, qui appartient d'ailleurs au genre Gigliolella Chabaud ef Choquet, 1954, a un nombre d'anneaux plus élevé que celui relevé chez nos spécimens. En ce qui concerne les deux autres espèces $A$. moniliformis et $A$. annulatus, elles présentent aussi un nombre d'anneaux' supérieur à 25.

En nous référant au travail d'HEYMONS (1935) sur les Pentastomidae, nous pensons pouvoir rapporter nos exemplaires à l'espèce Nettorhynchus (Armillifer) armillatus (Wyman, i 1835).

\section{N. armillatus dans les pays de l'ovest africain}

Ce parasite est très répandu dans tout l'ouesł africain. Ses formes adultes sont parasites des voies respiratoires de certains serpents. Elles ont été signalées parmi les ophidiens chez Python regius et $P$. sebae, ef parmi les vipéridés, chez Bitis arietans, B. gabonica, B. nasicornis et Cerastes cornutus.

Au point de vue hôtes intermédiaires, chez lesquels se trouvent les formes larvaires enkystées, un grand nombre d'espèces de mammifères ont été signalées. Nous ne citerons que les principales : le chien, le lion, le serval, le léopard, certains lémuriens et certains singes, l'oryctérope, le phacochère, certaines antilopes, des insectivores tels que le hérisson, des, viverridés, des rongeurs sauvages ef même la girafe. Dollfus, en 1958, a trouvé des kystes de cette espèce chez une grande roussette frugivore Eidolon helvum (Kerr, 1792) capturée à Bamako.

L'homme peut être aussi hôte intermédiaire 


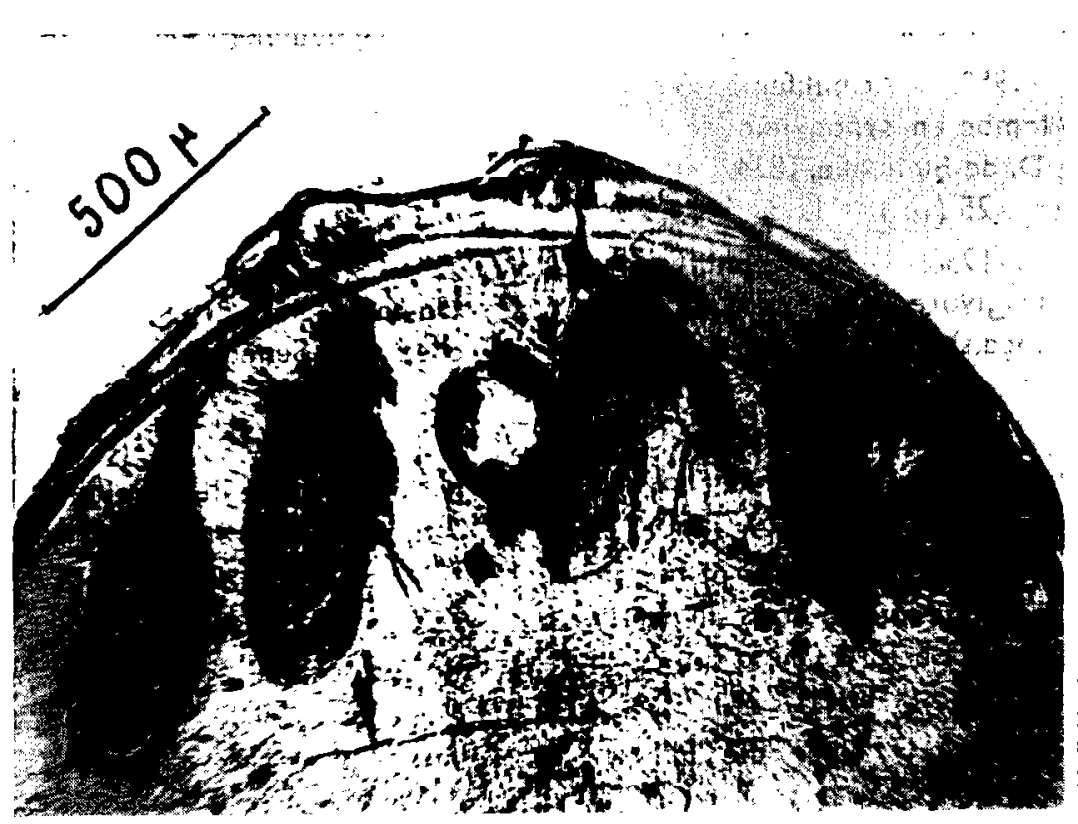

Fig. 3. - Extrémité céphalique de N. armillatus (nymphe).

et des cas de porocéphalose hépatique humaine ont déjà été signalés dans les pays de l'ovest africain (MONZIOLS, COLIGNON et ROY, 1920) (NOC et NOGUÉ, 1919). Quoique la présence des porocéphales dans l'organisme humain provoque souvent des troubles graves avec ictère pouvant aboutir à la mort, le diagnostic ne peut être posé que lors de l'autopsie.

\section{Cycle évolutif et mode de contamination}

Au point de vue cycle évolutif et modes d'infestation de l'hôte définitif et de l'hôte intermédiaire, les travaux de FÜLLEBORN en 1919, et ceux de NOC et CURASSON en 1920 ont montré que l'hôte intermédiaire s'infeste soit en avalant un serpent parasité présentant des œufs de pentastome dans sa cavité générale, soit en ce qui concerne les herbivores et les petits rongeurs par ingestion d'herbes ou de proies souillées par des excréments de serpents infectés.

- Le serpent hôte définitif s'infecte en avalant le mammifère porteur de kystes d'A. armillatus.

Dans le cas qui nous intéresse, il est peu probable que le chat se soit infesté en avalant un serpent. Nous pensons plutôt, soit à une contamination à partir d'aliments sovillés par des excréments de serpent, soit ce qui est encore fort pos- sible, à la suite de l'ingestion d'un petit rongeur lui-même porteur de formes larvaires de pentastomes. Dans le cas en effet où les formes larvaires sont ingérées par un hôte non favorable au développement des formes adultes, il peut se produire un phénomène de réenkystement ou de réencapsulement identique à celui que l'on constate chez certaines espèces d'helminthes.

Comme il est pratiquement impossible qu'un serpent dévore un chat, l'hébergement des formes larvaires chez cette espèce ne peut être qu'un cul de sac évolutif.

Nous avons signalé ce cas de porocéphalose féline, car à notre connaissance, le chat ne semble pas être un hôte intermédiaire habituel pour $N$. armillatus. Si l'infestation du chat domestique par des formes larvaires de pentastome a déjà été signalée en Chine par Faust en 1927, il s'agissuit de l'espèce Kiricepholus pattoni (Stephens. 1908).

Laboratoire central de l'Elevage. Dakar. Directeur : P. Mornet.

\section{BIBLIOGRAPHIE}

CHABAUD (A. G.) et CHOQUET (M. T.) 1954. - Nymphes du Pentastome Gigliolella (N. 
Gen.) brumpti (Giglioli, 1922) chez un Lémurien. Riv. parasit. Ital., $15: 331-6$.

DOLLFUS (R. P.) 1950. - Armillifer L. W. Sambon, 1922, tombe en synonymie de Nettorhynque $H$. D. de Blainville, 1824. Ann. Parasit. hum. comp. 25 (1-2) : 112-3.

DOLLFUS (R. P.) 1958, - Porocéphales d'un Chiroptère frugivore de Bamako sur Niger (Soudan Français). Bull. Mus., 2e Série, 30 (6) : 517-21.

FAUST (E. C.) 1927: - Linguatulids (Order Acarina) from man and other hosts in China. Amer. J. Trop. Med., 7 (5) : 311-25.

FAUST (E. C.) 1927. - Four species of lingatulids from man and other hosts in China. Abstract of Report before China Br. Amer. Soc. Parasit. J. Parasit. I3 (4) : 285.

FULLEBORN (F.) 1919. - Uber die Entwick- lung von Porocephalus und dessen pathogene Bedeutung. Beitr. z. Arch. f. Sch. Trop. Hyg. $23: 5-35$.

HEYMONS (R.) 1935: - Pentastomida Dr. H. $G$. Bronns Klassen und Ordnungen des Tierreichs Bd., V, Abt. IV, Buch I, pp. 1-268.

MONZIOLS, COLLIGNON ef ROY (J.) 1920, Un cas d'ictère grave suivi de mort et causé chez un Sénégalais par le Porocephalus armillatus C. R. Soc. Biol., 83 (2) : 28-9.

NOC (F.) et CURASSON (G.) 1920. - Contribution à l'étude de l'évolution biologique de Porocephalus armillatus Wyman. Bull. Soc. Poth, exot. $13(8): 656-9$.

NOC (F.) ef NOGUE 1919. - Note sur un cas de Porocéphalose. Bull. Soc. médico-rhirurg. franç. Ouest-Afric.; I (5) : 6-9 (Anal. in Bull. Inst. Pasteur, 1920, p. 288).

\section{SUMMARY}

Porocephalosis in a cat, due to Nettorhynchus (Armillifer) armillatus (Wyman, 1845).

The authors describe a case of porocephalosis in a destroyed cat detected at postmortem which had shown no symptoms while alive. About 50 cysts were noted situated fixed to the omentum, mesentry, the surface of the spleen and the liver, the abdominal wall, as also the mediastinum, diaphragm and thoracic wall. The cysts were eircumscribed by a thin and transparent conjunctive tissue reaction shell enclosing a larva, identified by the authors as that of Nettorhynchus (Armillifer) armillatus (Wyman 1845). The adult stage of this helminthis widespread in West Africa and parasitises the respiratory system of certain species of snakes. The authors recall the life-history of this parasite into which, the cat does not normally enter as an intermediate host.

\section{RESUMEN}

Porocefalosis por Nettorhynchus (Armillifer) armillatus (WYMAN, $1 ; 845$ ) sobre un gato.

Los autores nos presentan un caso de porocefalosis descubierto al practicar la autopsia sobre un gato que ellos sacrifican y que durante el periodo de observación no habia mostrado ningun síntoma.

Los quistes, unos cincuenta, se presentan fijados al epiplón; mesenterios, a la superficie del bazo y del hígado y también al peritoneo. Algunos se encuentran en cavidad torácica sobre mediastino y diafragma. Limitados por una membrana reaccional de naturaleza conjuntiva, delgada y transparente, encierra una larva que los autores identifican como de Nettorhynchus (Armillifer) armillatus (Wyman, 1.835).

El estado adulto de este verme, muy frecuente en Africa occidental, parasita las vias respiratorias de ciertas serpientes. Los autores recuerdan el ciclo evolutivo del parásito en el que el gato no parece ser un huesped intermediario habitual. 